\title{
Ketamine-associated nephropathy treated with renal transplantation: a case report
}

John SL Leung1', MB, BS, Vincent YK Poon'1, FRCSEd (Urol), Thomas YC Lam¹, FRCSEd (Urol), CK Chan', FRCSEd (Urol), Y Chiu', FRCSEd (Urol), TY Chu', FRCSEd (Urol), Samuel KS Fung ${ }^{2}$, FRCP (Edin), FRCP (Irel), WK Ma ${ }^{1}$ *, FRCSEd (Urol)

\author{
${ }^{1}$ Department of Surgery, Princess Margaret Hospital, Hong Kong \\ ${ }^{2}$ Department of Medicine and Geriatrics, Princess Margaret Hospital, Hong Kong
}

Hong Kong Med J 2021;27:219-21

* Corresponding author: drmawk@gmail.com

https://doi.org/10.12809/hkmj208588

\section{Case report}

We present a 37-year-old man who had been on continuous ambulatory peritoneal dialysis for 8 years owing to ketamine-associated end-stage renal failure. $\mathrm{He}$ received a cadaveric renal graft in December 2019 at Princess Margaret Hospital, Hong Kong.

He first presented with haematuria and dysuria in 2004. He had been abusing ketamine steadily for 4 years. Mid-stream urine culture and acid-fast bacillus culture were negative; urine cytology, renal function, and ultrasonography of the urinary system were unremarkable. The patient subsequently defaulted on investigations and follow-up appointments.

The patient returned in 2010 with more severe symptoms of ketamine cystitis and reflux nephropathy. At that time, he was taking 0.3 to $0.6 \mathrm{~g}$ of ketamine by nasal inhalation up to 10 times a day. He had urinary frequency every 10 minutes and creatinine was $283 \mu \mathrm{mol} / \mathrm{L}$ (estimated glomerular filtration rate [eGFR] $25 \mathrm{~mL} / \mathrm{min} / 1.73 \mathrm{~m}^{2}$ ). Flexible cystoscopy showed cystitis changes; a biopsy of the urothelium yielded neutrophilic exudates mixed with fibrin, and fibroblastic stromal reaction. Ultrasound scan revealed bilateral hydronephrosis and a thickened bladder wall. Non-contrast computed tomography showed thinning of the renal cortex and bilateral hydronephrosis as well as thickening of the ureters, all indicative of ureteric inflammation (Fig 1). Repeat urine cytology, routine culture, and acid-fast bacillus cultures were all negative.

Video cystometrogram demonstrated typical features of ketamine cystitis, namely that of a small and contracted bladder: the bladder capacity was $25 \mathrm{~mL}$; first desire to void was at $14 \mathrm{~mL}$; detrusor instability occurred at a Pdet of $22 \mathrm{cmH}_{2} \mathrm{O}$ (Fig 2). Additionally, bilateral grade III vesico-ureteric reflux was documented (Fig 1). The patient agreed only to a urethral catheter and refused upper tract urinary diversion with percutaneous nephrostomies.

He began abstaining from ketamine in 2010 but refused dialysis until 2011 when his creatinine had reached $1079 \mu \mathrm{mol} / \mathrm{L}$ (eGFR $5 \mathrm{~mL} / \mathrm{min} / 1.73 \mathrm{~m}^{2}$ ). Annual broad-spectrum drug screening of the patient's urine samples was negative for ketamine and its metabolites since then.

A repeat video cystometrogram in 2013, 3 years after complete abstinence from ketamine, showed improvements from his first video cystometrogram in 2010. Bladder capacity had improved to $124 \mathrm{~mL}$; first desire to void improved to $51 \mathrm{~mL}$. Detrusor
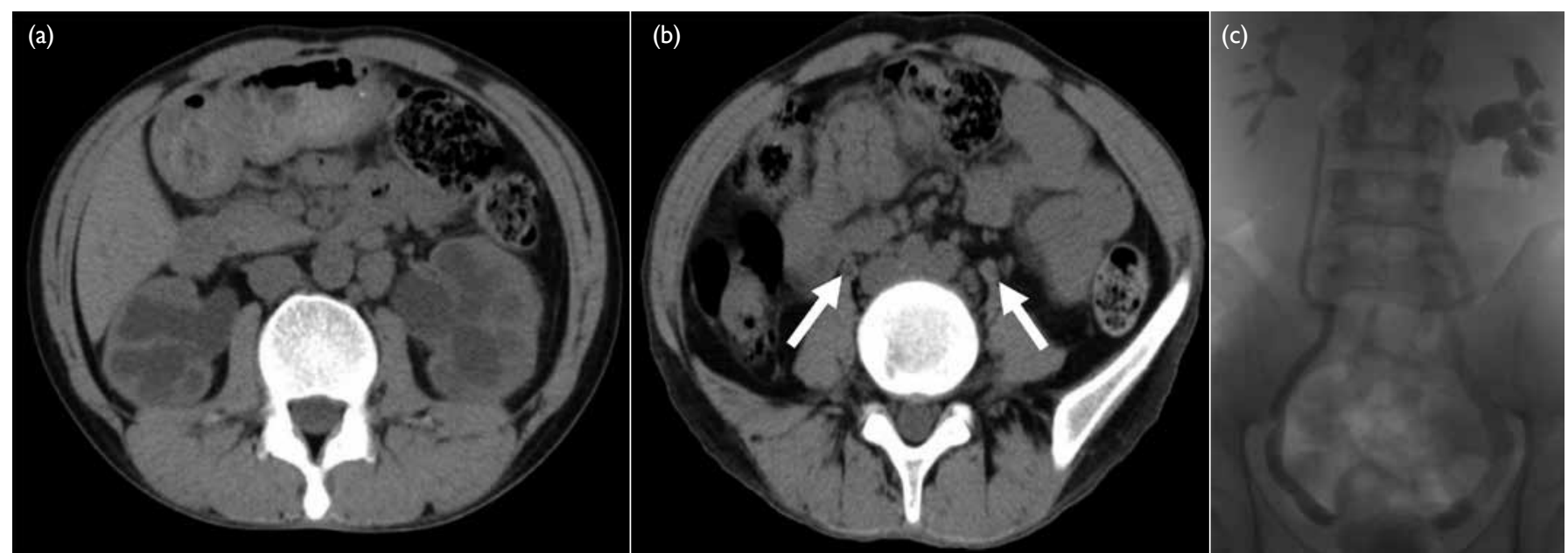

FIG I. A 37-year-old man with ketamine-associated end-stage renal failure. Non-contrast computed tomography film of the patient showing (a) bilateral hydronephrosis and hydroureters with thinning of the renal cortices and (b) bilateral thickening of the ureteric walls, more severe at the left ureter (white arrows). (c) Spot film from a video cystometrogram showing bilateral vesico-ureteric reflux with dilated ureters and a dilated left renal pelvis 
overactivity was noted at $120 \mathrm{~mL}$ when the Pdet was $30 \mathrm{cmH}_{2} \mathrm{O}$. Only a left grade I vesico-ureteric reflux was observed.

In 2019, another video cystometrogram showed improvement in the first desire to void to $75 \mathrm{~mL}$, no detrusor overactivity, and smooth bladder contour with no vesico-ureteric reflux (Fig 2). Bladder functional capacity was $200 \mathrm{~mL}$. It was therefore deemed worthwhile for him to undergo renal transplantation without augmentation cystoplasty.

The patient received a cadaveric renal graft from a 14-year-old donor. The operation was uneventful and he was no longer dialysis-dependent. At 8 weeks after transplantation, his creatinine level was $124 \mu \mathrm{mol} / \mathrm{L}\left(\mathrm{eGFR} 63 \mathrm{~mL} / \mathrm{min} / 1.73 \mathrm{~m}^{2}\right)$, and urine output about $2000 \mathrm{~mL}$ per day. At 12 weeks after the transplantation, his creatinine level was $122 \mu \mathrm{mol} / \mathrm{L}\left(\mathrm{eGFR} 65 \mathrm{~mL} / \mathrm{min} / 1.73 \mathrm{~m}^{2}\right.$ ), and urine output remained stable at about $2000 \mathrm{~mL}$ per day. Daytime frequency ranged from once every 1 to 3 hours, with 100 to $300 \mathrm{~mL}$ of urine per void. At 22 weeks after transplantation his creatinine level was $117 \mu \mathrm{mol} / \mathrm{L}$ (eGFR $68 \mathrm{~mL} / \mathrm{min} / 1.73 \mathrm{~m}^{2}$ ). Ultrasonography excluded graft kidney hydronephrosis and hydroureter.

\section{Discussion}

This is the first reported case of ketamine-associated nephropathy successfully treated with renal transplantation.

Ketamine cystitis was first reported in Hong Kong by Chu et $\mathrm{al}^{1}$ in 2007. Since then, numerous publications regarding its management have emerged. Abstinence remains the cornerstone of treatment as it results not only in improved cystitis symptoms, but also bladder capacity and compliance. $^{2,3}$ Early upper tract protection is paramount in patients with ketamine cystitis. Up to $16.8 \%$ of chronic ketamine abusers have unilateral or bilateral hydronephrosis owing to ureteric strictures or vesico-ureteric reflux. ${ }^{4}$ Strategies to protect the upper tract include percutaneous nephrostomy or long-term urethral catheterisation to keep the bladder decompressed. ${ }^{5}$ Internal ureteric stents may aggravate cystitis symptoms and hence may not be tolerated. ${ }^{6}$ In our case, the patient refused percutaneous nephrostomies for upper tract protection, and missed the window of opportunity to preserve his renal function before development of end-stage renal failure and need for dialysis.

There are two important prerequisites for

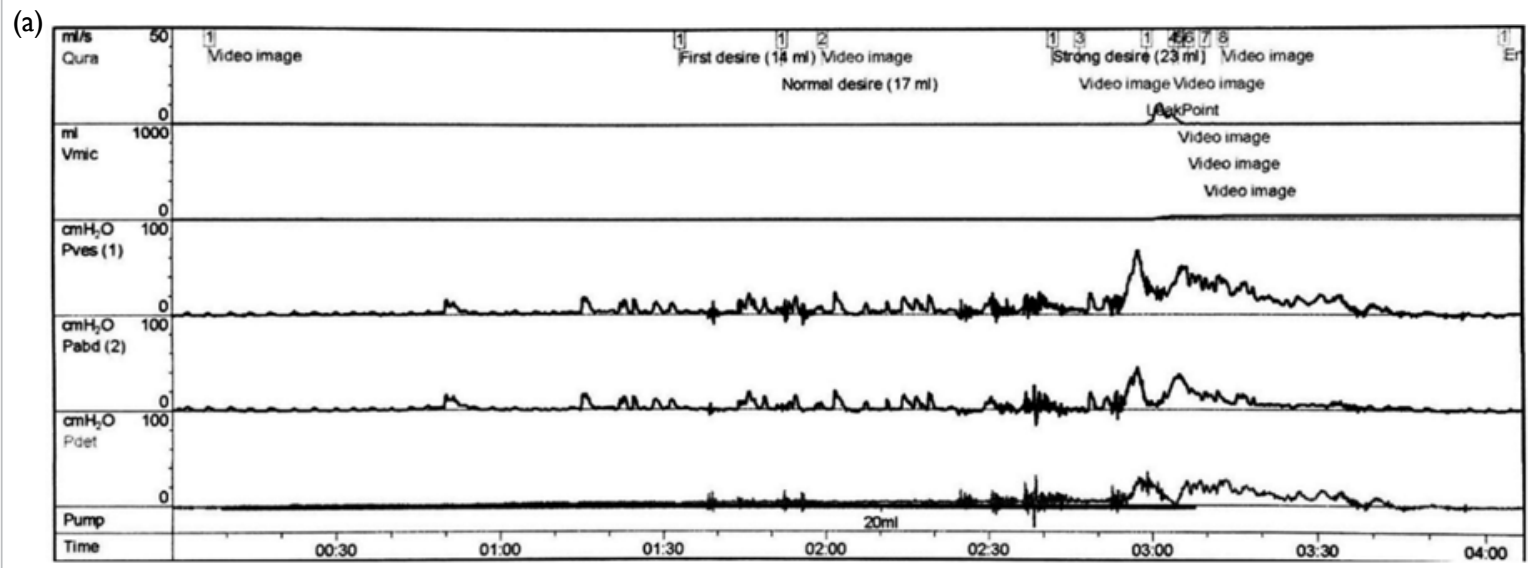

(b)

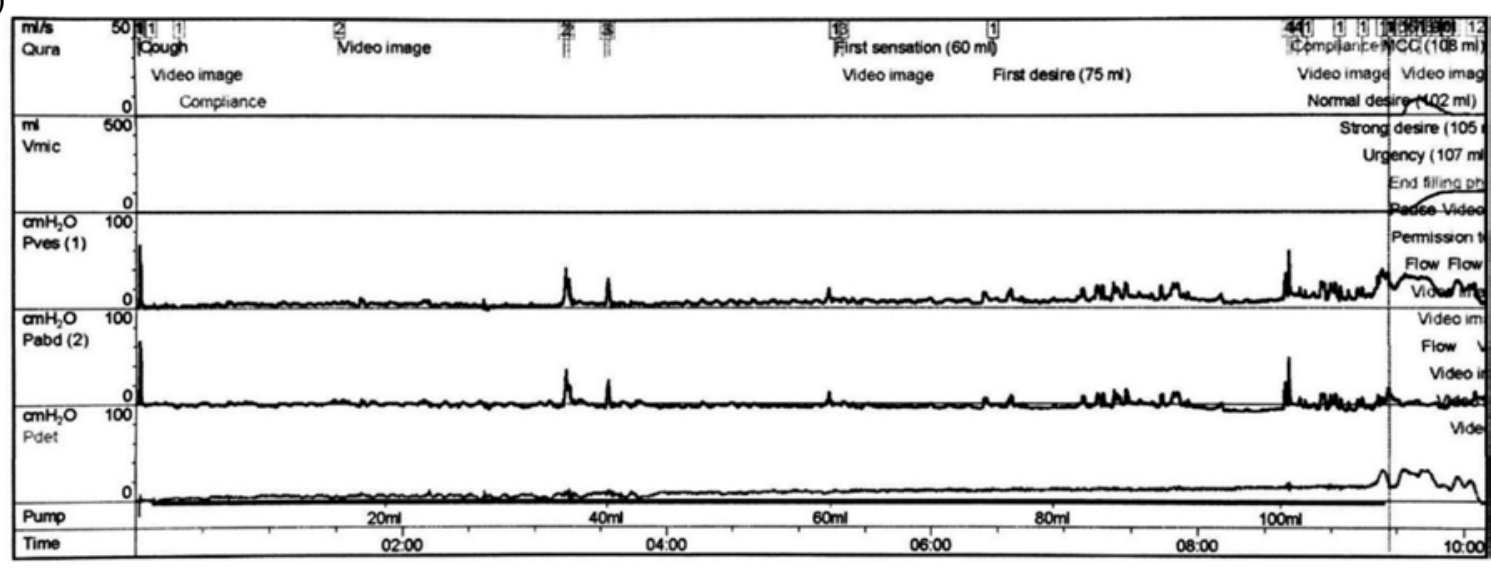

FIG 2. Same patient. (a) Cystometrogram tracing of the patient from 2010 showing an extremely non-compliant bladder with first desire to void at $14 \mathrm{~mL}$, and a bladder capacity of $25 \mathrm{~mL}$. (b) Repeat cystometrogram 10 months before transplantation (after 9 years of abstinence), showing improvements in compliance 
renal transplantation in ketamine-related endstage renal failure. The first is abstinence to ensure that the graft kidney and ureter are not subject to ketamine toxicity. Significant improvements in bladder compliance and capacity may be achieved only after at least 1 year of abstinence. ${ }^{1,3,7}$ Since improvement may take several years to stabilise, we suggest that consideration for transplantation should be at least 1 year after stabilisation of bladder function improvement and proven by serial negative urine toxicology screening. The second prerequisite is that bladder compliance and capacity are sufficient to accommodate the volume of urine produced by the graft kidney. A well-functioning graft kidney with a poorly compliant bladder can be damaged by vesico-ureteric reflux. Serial urodynamic studies to document improvements in bladder capacity before considering renal transplantation are mandatory. Although there is no absolute cut-off value for satisfactory bladder volume before transplantation, persistent vesico-ureteric reflux that does not resolve or downgrade with ketamine abstinence would be an indication for augmentation cystoplasty prior to transplantation. This will avoid debilitating urinary frequency after transplantation or early graft failure due to vesico-ureteric reflux. In our patient, bladder compliance and capacity improved steadily with prolonged abstinence, as documented by serial cystometrograms. Renal transplantation without augmentation cystoplasty was therefore an option. Patients with unsatisfactory bladder compliance and capacity should be counselled for augmentation cystoplasty before undergoing transplantation. This concept can be likened to the use of augmentation cystoplasty prior to renal transplantation in patients with high-pressure neurogenic bladder. ${ }^{8}$ Nonetheless augmentation cystoplasty for patients with ketamine cystitis is technically challenging owing to the fibrotic bladder with transmural thickening. Furthermore, the need for clean intermittent self-catheterisation afterwards may be cumbersome for this young patient group, especially if substantial ketaminerelated bladder pain remains.

Frequent follow-up after transplantation to monitor renal function, functional bladder capacity in the form of a bladder diary, and ultrasonography to exclude graft hydronephrosis should be maintained. A video cystometrogram to exclude vesico-ureteric reflux is mandatory should graft function deteriorate.

Early and sustained abstinence as well as advocation for early upper tract urinary diversion are important factors in the prevention of ketaminerelated nephropathy. Clinicians should maintain a low threshold of suspicion for ketamine abuse in young patients who present with recurrent lower urinary tract symptoms. ${ }^{9}$ A population-based survey of lower urinary tract symptoms in Hong Kong adolescents revealed that of those who reported lower urinary tract symptoms, $6.6 \%$ were substance abusers. ${ }^{10}$

Management of the symptoms of ketamine cystitis should adopt a stepwise approach starting with abstinence and analgesics; failing that, intravesical instillation of hyaluronic acid, hydrodistension, and eventually augmentation cystoplasty. ${ }^{3}$

\section{Author contributions}

All authors contributed to the concept or design of the study, acquisition of the data, analysis or interpretation of the data, drafting of the manuscript, and critical revision of the manuscript for important intellectual content. All authors had full access to the data, contributed to the study, approved the final version for publication, and take responsibility for its accuracy and integrity.

\section{Conflicts of interest}

The authors have no conflicts of interest to disclose.

\section{Funding/support}

This case report received no specific grant from any funding agency in the public, commercial, or not-for-profit sectors.

\section{Ethics approval}

The patient was treated in accordance with the Declaration of Helsinki and provided informed consent for the treatment/ procedures and consent for publication.

\section{References}

1. Chu PS, Kwok SC, Lam KM, et al. 'Street ketamine'associated bladder dysfunction: a report of ten cases. Hong Kong Med J 2007;13:311-3.

2. Cheung RY, Chan SS, Lee JH, Pang AW, Choy KW, Chung TK. Urinary symptoms and impaired quality of life in female ketamine users: persistence after cessation of use. Hong Kong Med J 2011;17:267-73.

3. Hong YL, Yee CH, Tam YH, Wong JH, Lai PT, Ng CF. Management of complications of ketamine abuse: 10 years' experience in Hong Kong. Hong Kong Med J 2018;24:175-81.

4. Yee $\mathrm{CH}$, Teoh JY, Lai PT, et al. The risk of upper urinary tract involvement in patients with ketamine-associated uropathy. Int Neurourol J 2017;21:128-32.

5. Chu P, Ma WK, Wong S, et al. The destruction of the lower urinary tract by ketamine abuse: a new syndrome? BJU Int 2008;102:1616-22.

6. Tsai YC, Kuo HC. Ketamine cystitis: Its urological impact and management. Urol Sci 2015;26:153-7.

7. Yee CH, Lai PT, Lee WM, Tam YH, Ng CF. Clinical outcome of a prospective case series of patients with ketamine cystitis who underwent standardized treatment protocol. Urology 2015;86:236-43.

8. Basiri A, Otookesh H, Hosseini R, Moghaddam SM. Kidney transplantation before or after augmentation cystoplasty in children with high-pressure neurogenic bladder. BJU Int 2009;103:86-8.

9. Ng SH, Tse ML, Ng HW, Lau FL. Emergency department presentation of ketamine abusers in Hong Kong: a review of 233 cases. Hong Kong Med J 2010;16:6-11.

10. Tam YH, Ng CF, Wong YS, et al. Population-based survey of the prevalence of lower urinary tract symptoms in adolescents with and without psychotropic substance abuse. Hong Kong Med J 2016;22:454-63. 